\title{
不同条件下胰岛素别构中间体 $\left(\mathbf{T}_{\mathbf{3}} \mathbf{R}_{\mathbf{3}}\right)$ 的初步晶体学分析
}

\author{
艾析 (1) 曾宗浩 (1) 黄培堂 (2) 王大成 ${ }^{(1) * ~}$
}

(1)中国科学院生物物理研究所, 北京 100101; (2)军事医学科学院生物工程研究所, 北京 100071。*联系人)

\section{关键词费鴡素 $\mathbf{T}_{3} \mathbf{R}_{3}$ 结构 效应物 结晶 初步晶体学分析}

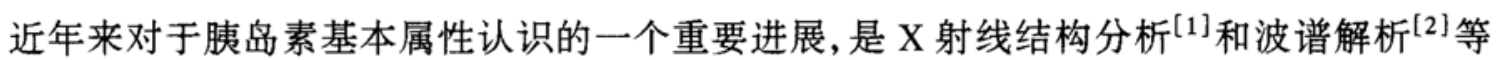
揭示它具有经典别构蛋白的特性. 已发现其单体具有 $T, R\left(\right.$ 或 $R^{f}$ ) 构象, 其六聚体则表现出 3 种构象态: $T_{6}, T_{3} R_{3}$ (或 $T_{3} R_{3}^{f}$ ) 及 $R_{3}{ }^{[3]}$. 胰岛素 $T$ 结构与 $R$ 结构的区别在于: $B$ 链的 B1-B8 在

被磷酸化后可结合 STAT5(Signal Transducer and Activator of Transcription 5), 从而使结合于 $\gamma$ 胞内域的 JAK3 磷酸化 STAT5, 磷酸化的 STAT5 可形成同源二聚体, 转位到细胞核内开动 基因表达 ${ }^{[6]}$. 本文发现 JAK3 与 c-myc 基因被诱导有关系, 不仅有助于说明 c-myc 基因被诱导 的机制, 而且揭示了 IL-2 的 4 条信号途径中的 JAK-STAT 途径与 c-myc 途径通过 JAK3 进行 crosstalk, 为进一步研究 IL-2 信号转导途径之间的网络联系打下了基础.

虽然用全长 JAK3 替代 $\gamma$ 亚基胞内域也能诱导 c-myc 基因的表达(数据未给), 但本结果 表明只要有 JAK3 催化功能域就可完成这一过程, 即这个功能域本身足以活化某些信号分 子, 从而诱发活化 c-myc 基因的胞内信号级联反应. 那么 JAK3 的 $\mathrm{N}$ 端起什么作用呢? 我们 通过醉母双杂交系统证实了 JAK3 的 $\mathrm{N}$ 端可以与 IL-2R $\gamma$ 胞内域结合, 从而使 JAK3 针定在 IL-2R $\gamma$ 上(待发表). 此外, 我们通过双杂交系统篣选到一个与 JAK3 的 N 端相互作用的蛋白 质, 说明 JAK3 的 N 端可能在底物识别中起作用(待发表), 这些工作将为 JAK3 新功能的发现 打下基础.

\section{参考文 献}

1 Minami Y, Kono T, Miyazaki T, et al. The IL-2 receptor complex: Its structure, function, and target genes. Annu Rev Immunol, 1993, 11: 245

2 Nakamura Y, Russell S M, Mess S A, et al. Heterodimerization of the IL-2 receptor $\beta$ - and $\gamma$-chain cytoplasmic domains is required for signalling. Nature, 1994, 369: 330 333

3 Johnston J A, Kawamura M, Kirken R A, et al. Phosphorylation and activation of the Jak-3 Janus kinase in response to interleukin-2. Nature, 1994, 370: 151 153

4 Sambrook J, Fritsch E F, Mamiatis T, et al. Molecular Cloning: A Laboratory Manual. New York: Cold Spring Harbor Laboratory Press, 1989

5 Minamoto S, Mori H, Hatakeyama M, et al. Characterization of the heterodimeric complex of human IL-2 receptor $\alpha, \beta$ chains reconstituted in a mouse fibroblast cell line, L929. J Immunol, 1990, 145: 2 177 2 182

6 Gaffen S L, Lai S Y, Xu W, et al. Signaling through the interleukin- 2 receptor $\beta$ chain activates a STAT-5-like DNA-binding activity. Proc Natl Acad Sci USA, 1995, 92: 7 192 7 196

（1997-05-27 收稿, 1997-07-22 收修改稿） 
前者为伸展状态, 在后者则为螺旋状态; 相应地, $\mathrm{T}$ 结构六聚体中, 轴上 $\mathrm{Zn}^{2+}$ 的配位为六配位, 而在 $R$ 结构六聚体中为四配位. 随后发现的 $R^{f}$ 结构 ${ }^{[4]}$ 的特点为: B1-B3 仍为伸展状态, 而 B4$B 19$ 为螺旋区. $\mathrm{R}$ 结构最初发现存在于长效胰岛素中, 它可延长胰岛素六聚体解聚为单体的 时间, 从而延长其药效 ${ }^{[1]}$. 人们认为 $\mathrm{R}$ 结构的产生可能和胰岛素与其受体的结合有关 ${ }^{[5]}$. 由 于迄今为止只有少数别构蛋白体系的精确三维结构得到阐明, 因此对胰岛素这一别构现象的 精细研究无疑对深入了解别构蛋白的分子机理具有重要意义.

已经通过 X 射线衍射分析 ${ }^{[1,4,6-8]}$ 、核磁共振波谱 $(N M R)^{[2,5,9]}$ 及其他波谱学方法 ${ }^{[5,9]}$ 发 现, 苯酚及其衍生物 (环己醇、4-硝基苯酚、4-羟甲基苯、3-甲基苯酚、间苯二酚、3-三氟甲基苯酚 等 $)^{[1,5,6]}$ 可诱导产生胰岛素六聚体的 $\mathrm{R}_{6}$ 结构, 高浓度 $\mathrm{Cl}^{-}, \mathrm{SCN}^{-}, \mathrm{OSN}^{-[2,6,8,9]}$ 或较低浓 度的苯酚 ${ }^{[6]}$ 及其难溶衍生物 (4-差基苯胺、 $4^{\prime}$ - 差基乙酰苯胺等) ${ }^{[4,7]}$ 则可以诱导产生 $T_{3} R_{3}$ (或 $T_{3} R_{3}^{f}$ ) 结构. 在这一别构体系中, $T_{3} R_{3}$ 可视为一中间态, 对它的详细研究具有特殊价值. 此前 的研究表明, 只有高浓度盐离子和苯酚衍生物可诱导此种结构, 如上所述. 我们在研究胰岛素 突变体时发现, 实际上这类结构的效应物不限于上述两类化学因素 ${ }^{[10]}$. 在此基础上, 我们运 用 $\mathrm{rDNA}$ 重组天然人胰岛素结晶体系, 系统搜索了不同有机小分子对诱导产生 $\mathrm{T}_{3} \mathrm{R}_{3}$ 结构的作 用. 结果表明, 在含有不同有机小分子的结晶体系中, 均可产生具有 $T_{3} R_{3}$ 结构类型的结晶, 显示可诱导 $T_{3} R_{3}$ 结构产生的效应物具有多样性. 本文报道一系列具有 $T_{3} R_{3}$ 结构类型的天 然人胰岛素及其突变体的结晶和初步晶体学分析.

\section{1 实验与结果}

\section{1 材料与结晶}

$\mathrm{rDNA}$ 重组天然人胰岛素(以下简称 $\mathrm{rDNA}-\mathrm{HI}$ ), $\mathrm{A} 21^{\text {Ser }}$ 人胰岛素突变体 (以下简称 $\mathrm{A} 21 \mathrm{~S}$ $\mathrm{HI}$ ), $\mathrm{A} 21^{\mathrm{Ser}}{ }_{-} \mathrm{B} 27^{\mathrm{Arg}}-\mathrm{B} 30^{\mathrm{Thr}}{ }_{-} \mathrm{NH}_{2}$ 人胰岛素突变体 (以下简称 $\mathrm{SRN}-\mathrm{HI}$ ) : 均由丹麦 Novo 研究所提 供; Tris 为 Sigma 公司产品; 其余试剂均为分析纯试剂, 国内生产.

对 rDNA-HI 的研究, 首先在含 $\mathrm{Zn}^{2+}$ 体系中进行. 结晶试验采用 Citrate-Tris- $Z n A_{2}$ 作为 基本条件, 然后分别在池液中加入有机分子二氧六环(Dioxane)、异丙醇( Isopropanol)及二甲基 甲酰胺(DMF), 构成 3 种体系 (见表 1 中的 $1,2,3$ ). 在此基础上, 用含 Dioxane 的体系, 分别在 悬滴中再加入 Isopropanol 和 DMF, 构成两种复合体系 (见表 1 中的 4,5). 结晶采用悬滴汽相

表 1 “在一些有机分子存在下 rDNA-HI 及其突变体的结晶条件

\begin{tabular}{|c|c|c|c|c|c|c|c|c|}
\hline \multirow{2}{*}{ 样品 } & \multicolumn{5}{|c|}{ rDNA-HI } & \multicolumn{3}{|c|}{ 突变体 } \\
\hline & 1 & 2 & 3 & 4 & 5 & 6 & A21S-HI & SRN-HI \\
\hline $\mathrm{A}$ 液 & \multicolumn{5}{|c|}{$12 \mathrm{mg} / \mathrm{mL}$ 蛋白溶于 $0.03 \mathrm{HCl}$} & $\begin{array}{l}18 \mathrm{mg} / \mathrm{mL} \text { 蛋白溶 } \\
\text { 千 } 0.03 \mathrm{~mol} / \mathrm{L} \mathrm{HCl}\end{array}$ & $\begin{array}{l}10 \mathrm{mg} / \mathrm{mL} \text { 蛋白溶 } \\
\text { 于 } 0.03 \mathrm{~mol} / \mathrm{L} \mathrm{HCl}\end{array}$ & $\begin{array}{l}10 \mathrm{mg} / \mathrm{mL} \text { 蛋白浴 } \\
\text { 于 } 0.03 \mathrm{~mol} / \mathrm{L} \mathrm{HCl}\end{array}$ \\
\hline \multirow[t]{5}{*}{$\mathrm{B}$ 液 } & \multirow{2}{*}{\multicolumn{5}{|c|}{$0.01 \mathrm{~mol} / \mathrm{L}$ 柠檬酸 }} & $2 \% \mathrm{NaH}_{2} \mathrm{PO}_{4}$ & $0.01 \mathrm{~mol} / \mathrm{L}$ 柠㮠酸 & $2 \% \mathrm{NaH}_{2} \mathrm{PO}_{4}$ \\
\hline & & & & & & $8 \%$ MPD & $6 \%$ Tris & $15 \%$ MPD \\
\hline & \multirow{3}{*}{\multicolumn{5}{|c|}{$0.02 \% \mathrm{ZnAc}_{2}$}} & $15 \%$ DMF & $0.01 \% \mathrm{ZnAc_{2 }}$ & $10 \% \mathrm{DMF}$ \\
\hline & & & & & & $6 \%$ Tris & & $6 \%$ Tris \\
\hline & & & & & & $\mathrm{pH} 7.57$ & $\mathrm{pH} 9.25$ & $\mathrm{pH} 8.10$ \\
\hline 悬滴 & $A+B$ & $A+B$ & $A+B$ & $A+B+$ 异丙醇 & $\mathrm{A}+\mathrm{B}+\mathrm{DMF}$ & & $A+B$ & \\
\hline 池液 & B + 1.4-Diox & $\mathrm{B}+$ 异丙醇 & $\mathrm{B}+\mathrm{DMF}$ & B+1.4-Diox & B+1.4-Diox & & B+1,4-Diox & \\
\hline 静眐液滴 & & & & & & $A+B$ & & $A+B$ \\
\hline 温度") & $14 \sim 23 \mathrm{C}$ & $14 \sim 23 \mathrm{C}$ & $14 \sim 23 \mathrm{C}$ & $14 \sim 23 \mathrm{C}$ & $14 \sim 23 \mathrm{C}$ & $14 \mathrm{C}$ & $14 \sim 23 \mathrm{C}$ & $14 \mathrm{C}$ \\
\hline
\end{tabular}

a) 培养盒首先盈于 $14 \mathrm{C}$, 出现晶核后移至室温 
扩散法, 各项最佳晶体生长条件经多次实验摸索确定, 详列于表 1 中. 经 $4 \sim 6$ 周, 上述 5 个体 系均可长出优质单晶体, 最大棱边的长度均可大于 $0.5 \mathrm{~mm}$, 从外形可清楚分辨为三方晶型 (见图 1).
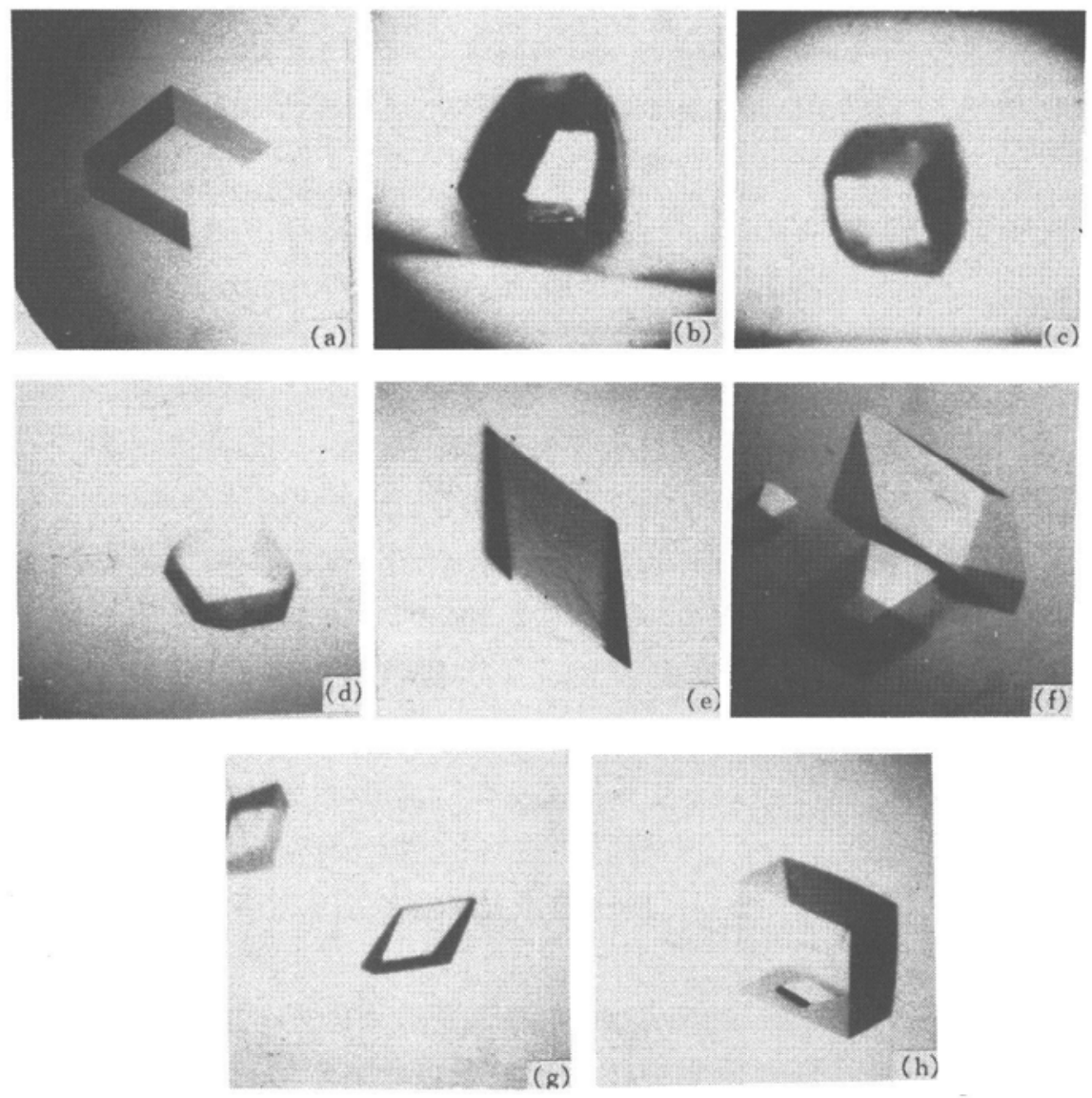

图 1 在不同结晶体系中获得的 rDNA-HI 及其突变体的单晶体

(a) rDNA-HI(二氧六环); (b) rDNA-HI(异丙醇); (c) rDNA-HI(DMF); (d) rDNA-HI(二氧六环＋异丙醇);

(e) rDNA-HI(二氧六环 + DMF); (f) rDNA-HI(无锌); (g) A21S-HI(二氧六环); (h) SRN-HI(无锌)

与此同时, 还进行了无 $\mathrm{Zn}^{2+}$ 结晶试验. 采用 $\mathrm{NaH}_{2} \mathrm{PO}_{4}$-Tris 缓冲体系, 在加入 DMF 和 MPD 的条件下 (见表 1 中的 6), 以微量静置法获得优质三方晶型单晶体(图 1(f)).

与上述天然 rDNA-HI 相平行, 对两种人胰岛素突变体 A21S-HI 和 SRN-HI 也进行了结晶 试验. 在含 Dioxane 的体系(表 1) 中获得了 A21S-HI 的含 $\mathrm{Zn}^{2+}$ 三方晶体; 在无 $\mathrm{Zn}^{2+}$ 体系 (表 1 ) 中, 获得 SRN-HI 的三方晶体 (参见图 $1(\mathrm{~g}),(\mathrm{h})$ ). 但在它们的最佳晶体生长条件中, $\mathrm{pH}$ 值 都比在 rDNA-HI 中更偏碱性(见表 1).

\section{2 初步晶体学分析}

在 Mar Research Image Plate 面探测仪上测得 6 颗天然人胰岛素晶体及两颗人胰岛素突 变体晶体的晶胞参数及空间群, 详列于表 2 . 作为参照, 表 2 同时给出具有 $T_{3} R_{3}$ 结构 $(4 Z n$ 型 $)^{[1]}$ 和 $T_{6}$ 结构 $(2 \mathrm{Zn} \text { 型 })^{[1]}$ 三方胰岛素的基本晶体学参数, 从中可见, 这 8 颗晶体的基本晶体 学参数与具有 $T_{3} R_{3}$ 结构的晶型基本相同, 表明它们应属于 $T_{3} R_{3}$ 结构. 
表 2 在含不同有机分子结晶体系中获得的 rDNA-HI 及其突变体单晶的基本晶体学数据

\begin{tabular}{|c|c|c|c|c|c|c|c|c|c|c|}
\hline 晶体 & \multicolumn{6}{|c|}{ rDNA - HI } & \multicolumn{2}{|c|}{ 突变体 } & $\begin{array}{l}4 \mathrm{Zn}^{[1]} \\
\left(\mathrm{T}_{3} \mathrm{R}_{3}\right)\end{array}$ & $\begin{array}{c}2 \mathrm{Zn}^{[1]} \\
\left(\mathrm{T}_{6}\right)\end{array}$ \\
\hline 空间群 & $\mathrm{R} 3$ & $\mathrm{R} 3$ & $\mathrm{R} 3$ & R3 & R3 & R3 & R3 & R3 & R3 & R3 \\
\hline$($ 六方格子 $) \mathrm{c}^{=}$ & 37.84 & 38.24 & 37.72 & 38.27 & 38.58 & 39.22 & 38.85 & 37.98 & 37.6 & 34.0 \\
\hline
\end{tabular}

衍射数据的初步分析表明, 除 SRN-HI 无 $\mathrm{Zn}^{2+}$ 晶体外, 其余晶体的分辨率均优于 0.20 $n m$. 目前, 已在装有 X-200B 型面探的 X 射线衍射仪上(SIEMENS), 收集了分别含 Dioxane, DMF, Isopropanol 的 rDNA-HI 晶体(表 1,2 中的样品 $1,2,3$ 和 6) 的 $0.19 \mathrm{~nm}$ 分辨率衍射数 据, 并用 XENGEN 软件包进行了处理. 数据的 $R_{\text {merge }}$ 在 $2.93 \% \sim 7.38 \%$ 之间, 表明质量很好.

\section{2 讨论}

在目前已知的胰岛素三方晶体中, 只有 3 种晶型, 它们的空间群相同 (R3), 但晶胞参数显 著不同 ${ }^{[1]}$ : (1) $a_{\mathrm{H}}=82.5 \times 10^{-1} \mathrm{~nm}, c_{\mathrm{H}}=34.0 \times 10^{-1} \mathrm{~nm}$; (2) $a_{\mathrm{H}}=80.7 \times 10^{-1} \mathrm{~nm}, c_{\mathrm{H}}=$ $37.6 \times 10^{-1} \mathrm{~nm}$; (3) $a_{\mathrm{H}}=79.9 \times 10^{-1} \mathrm{~nm}, c_{\mathrm{H}}=40.4 \times 10^{-1} \mathrm{~nm}$. 精确的结构解析证明, 它们 分别相应于胰岛素的 $T_{6}, T_{3} R_{3}$ 和 $R_{6}$ 结构. 本文报道的所有结晶的晶胞参数, 与 (2)相比较, 在 $a, b$ 方向相差 $0.3 \% \sim 1 \%$ 以内, 在 $c$ 方向相差 $1.5 \%$ 以内, 基本上是同晶的, 表明它们的晶 体结构均属于 $\mathrm{T}_{3} \mathrm{R}_{3}$ 类型.

此前, 只有高浓度 $\mathrm{Cl}^{-}$和 $\mathrm{SCN}^{-}$及低溶解度苯酚衍生物诱导出胰岛素 $\mathrm{T}_{3} \mathrm{R}_{3}$ 晶体结构的报 道 ${ }^{[1,4 \sim 6]}$; 同时波谱分析显示, $\mathrm{SCN}^{-}$, $\mathrm{OSN}^{-}$等也可能诱导此类结构在溶液中产生 ${ }^{[8]}$. 同时, $\mathrm{R}$ 类型的结构与酚基存在有关. 在本文报道的结晶体系中, 既无高浓度的无机离子, 亦无酚基 类似物存在, 但却肯定地获得了 $T_{3} R_{3}$ 类型结构的结晶, 表明诱导 $T_{3} R_{3}$ 结构的效应物具有多 样性. 此外, 除 $\mathrm{N}$ 端的螺旋和伸展构象的差别之外, $\mathrm{Zn}^{2+}$ 在 $\mathrm{T}$ 结构中取六配位, 在 $\mathrm{R}$ 结构中 取四配位, 也被认为是这类结构的重要特征. 本报道的结果证实, 在无 $\mathrm{Zn}^{2+}$ 条件下, 也获得了 $\mathrm{T}_{3} \mathrm{R}_{3}$ 结构类型的结晶(参见表 1,2), 表明特殊的金属离子配位并非 $\mathrm{T}_{3} \mathrm{R}_{3}$ 结构的基本特征, 这 与波谱分析对溶液体系的研究结果 ${ }^{[9]}$ 一致. 上述结果对探讨 $T_{3} R_{3}$ 结构的体内发生及其可能 的生物学作用很有意义.

在目前的结晶体系中, 最有可能诱导 $\mathrm{T}_{3} \mathrm{R}_{3}$ 结构产生的是一系列有机小分子: Dioxane, Isopropanol 和 DMF. 当然, 也不排除其他因素, 如 $\mathrm{pH}$ 、缓冲剂的可能影响. 明确的结果有待 这一系列晶体结构的测定. 目前,这些晶体结构正在逐个解析之中.

致谢 本工作为国家“八六三”高技术研究计划资助项目。

\section{参考文献}

1 Derewenda U, Derewenda Z, Dodson E J, et al. Phenol stabilizes more helix in a new symmetrical zinc insulin hexamer. Nature, 1989, 338(13): 594 596

2 Kaarsholm N C, Ko H C, Dunn M F. Comparison of solution structural flexibility and zinc binding domains for insulin, proinsulin, and miniproinsulin. Biochemistry, 1989, 28(10): $4427 \sim 4435$

3 Brader M L, Dunn M F. Insulin hexamer: new conformations and applications. TIBS, 16: 341 345 


\title{
微激光的量子模式理论
}

\author{
刘仁红 ${ }^{(1)}$ 谭维翰 $^{(2)}$ 许文沦 (2) 张菊芳 (2)
}

(1)中国科学院上海光学精密机械研究所, 上海 201800; (2)上海大学物理系, 上海 201800)

\section{关键词量子模式 闵值条件}

在激光发展中开腔模式理论起了很重要的作用. 激光开腔模式理论是一种半经典理论. 激活介质中的原子用量子力学描述, 而辐射场则服从经典的 Maxwell 方程, 场的量子化已被忽 略了. 故开腔模式理论也未能给出激光模式中光子的统计分布. 只是后来的全量子激光理论 才证明了, 由于原子的自发辐射,腔的损耗, 以及作为光原的激活原子的无规注入, 在阈值上的 单模激光光子服从 Poisson 分布, 其量子噪音满足 $\left\langle(\Delta n)^{2}\right\rangle=\langle n\rangle$. 有不少作者 ${ }^{[1 ~ 6] ~}$ 证明采用 规则的原子注入能减少激光噪音到低于 Poisson 分布, $\left\langle(\Delta n)^{2}\right\rangle\langle\langle n\rangle$, 即亚 Poisson 分布. 基 于规则原子注入模型, 可导出量子化的辐射场的密度矩阵 $\rho$ 的主方程 ${ }^{[2]}$. 对 Laser 情形, 可解 析求得主方程的稳态解 ${ }^{[5]}$. 但在 Maser 情形, 这些稳态解中有时表现出非物理的 “负几率分 布” [3]. 另一方面, 无损耗腔的量子模式理论表明, 当满足条件 $g \tau \sqrt{\pi}=q \pi, q$ 为整数, 存在一 种瓶颈态 ${ }^{[7,8]}$. 对有损耗膑, 辐射场的密度矩阵变化一般采用分步表示, 即 $\rho_{f}\left(t_{i+1}\right)=\exp$ $\left(L t_{p}\right) F\left(t_{i n}\right) \rho_{f}\left(t_{i}\right)^{[7 \sim 9]}$. 式中 $\rho_{f}\left(t_{i}\right)$ 代表场的初始分布, $t_{i n}$ 为注入原子在腔内停留并与其相 互作用的时间, $F\left(t_{i n}\right)$ 代表相互作用的影响; $t_{p}$ 为原子已飞出腔, 仅有损耗 $\exp \left(L t_{p}\right)$, 且 $t_{p} \gg$ $t_{i n}, t_{p}=t_{i+1}-t_{i}-t_{i n} \simeq t_{i+1}-t_{i}$. 在原子与场相互作用时间 $t_{i n}$ 内, 腔的损耗已被忽略掉, 这 就是分步模式理论. 故分步模式是一种 $t_{p} \gg t_{i n}$, 忽略在 $t_{i n}$ 时间的损耗的近似的模式理论. 若 在 $t_{\text {in }}$ 内损耗较大, 这种近似模式理论也是不适用的. 它与主方程的关系为: 若假定 I ) 粗粒

4 Smith G D, Ciszak E, Pangborn W. A novel complex of a phenolic drivative with insulin: Structural features related to the T $\rightarrow R$ transition. Protein Science, 1996, 5(8): $1502 \sim 1511$

5 Brader M L, Kaarsholm N C, lee R W K, et al. Characterization of the R-state insulin hexamer and its derivatives: the hexamer is stabilized by heterotropic ligand binding interactions. Biochemistry, 1991, 30(27): $6636 \sim 6645$

6 Whittingham J L, Chaudhuri S, Eleanor J D, et al. X-ray crystallographic studies on hexameric insulins in the presence of helix-stabilizing agents, thiocyanate, methylparaben, and phenol. Biochemistry, 1995, 34(47): 15 553 15 563

7 Smith G D, Ciszak E. The structure of a complex of hexameric insulin and 4 '-hydroxyacetanilide. Proc Natl Acad Sci USA, 1994, 91(19): $8851 \sim 8855$

8 Ciszak E, Smith G D. Crystallographic evidence for dual coordination around zinc in the $T_{3} R_{3}$ human insulin hexamer. Biochemistry, 1994, 33(6): 1512 1 1517

9 Roy M, Brader M L, Lee R W K, et al. Spectroscopic signatures of the T to R conformational transtion in the insulin hexamer. J Biol Chem, 1989, 2(32): $19081 \sim 19085$

10 Wang D C, Zeng Z H, Hu Y L, et al. A new structural type of zinc insulin observed in a mutant of [A21-Ser]-human insulin. In: Du Y C, Tam J P. Zhang Y S eds. Peptide, Biology and Chemistry, Proceeding of the 1992 Chinese Peptide Symposium, Leiden, 1992. Leiden: ESCOM, 1993, 241 244 